\title{
Computational Modeling of the Interaction of Silver Clusters with Carbohydrates
}

Felipe E. Gallegos ${ }^{1}$, Lorena M. Meneses ${ }^{1 *}$, Sebastián A. Cuesta ${ }^{1}$, Juan C. Santos ${ }^{2}$, Josefa Arias $^{1}$, Pamela Carrillo ${ }^{3}$, María F. Pilaquinga ${ }^{4}$

1 Laboratory of Computational Chemistry, Chemical Science Department, Pontificia Universidad Católica del Ecuador, Quito, Ecuador

${ }^{2}$ Ingeniería G-Mar LTDA, Avenida Hacienda Macul 6370, Peñalolén, Santiago, Chile

${ }^{3}$ Chemistry Department, University of Liverpool, Liverpool, United Kingdom.

${ }^{4}$ Laboratory of Nanotechnology, Chemical Sciences Department, Pontificia Universidad Católica del Ecuador, Quito, Ecuador 


\section{Supporting Information}

Table S1. Energies $(\mathrm{kcal} / \mathrm{mol})$ of all tested silver clusters compared to the most stable conformation in each system. (D) and (C) represent the most stable conformations for the three-silver-atom cluster and five-silver-atom cluster, respectively.

\begin{tabular}{|c|c|c|}
\hline \multirow{2}{*}{ Conformation } & \multicolumn{2}{|c|}{ E (kcal/mol) } \\
\hline & $3 \mathrm{Ag}$ & $5 \mathrm{Ag}$ \\
\hline (A) & 3.50 & 5.72 \\
\hline (B) & 1.82 & 8.20 \\
\hline (C) & 0.45 & 0.00 \\
\hline (D) & 0.00 & 13.90 \\
\hline
\end{tabular}
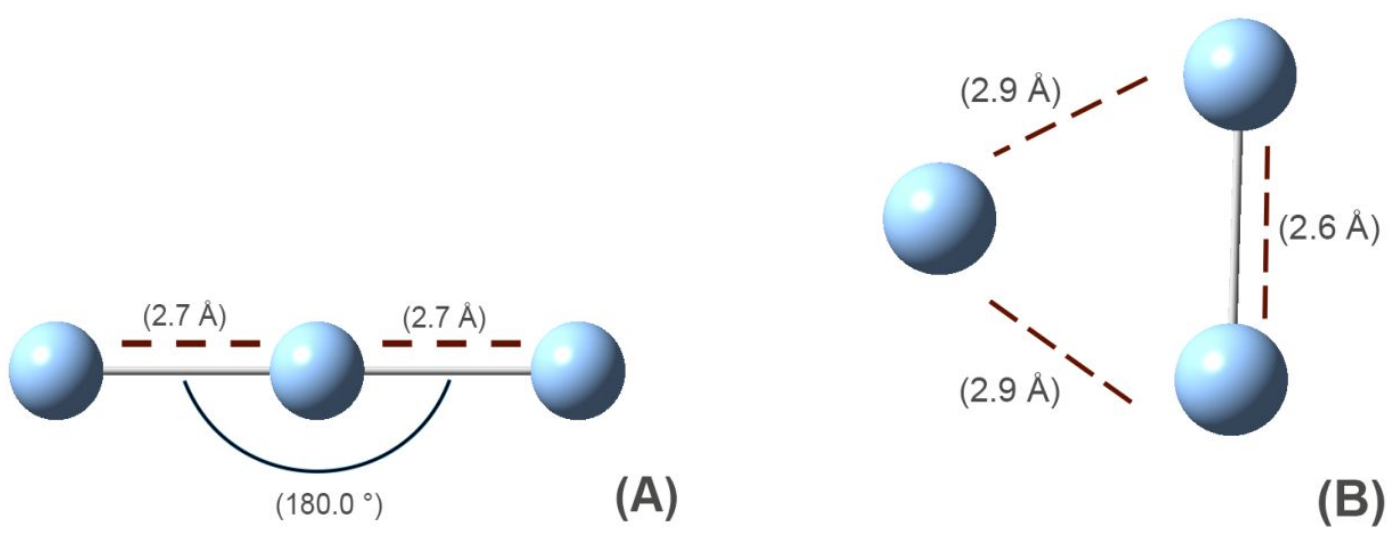

(A)

(B) 

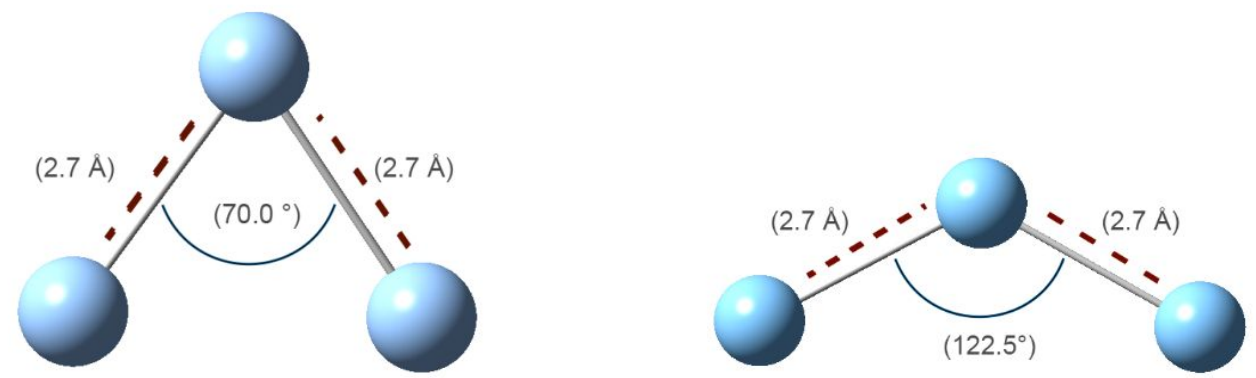

(C)

(D)

Figure S1. All silver conformations converged for the three-silver-atom cluster. (A) a linear conformation with a $180^{\circ}$ degrees angle, (B) an opened shape conformation, (C) and (D) planar conformations types. (D) represents the most stable conformation in this study.
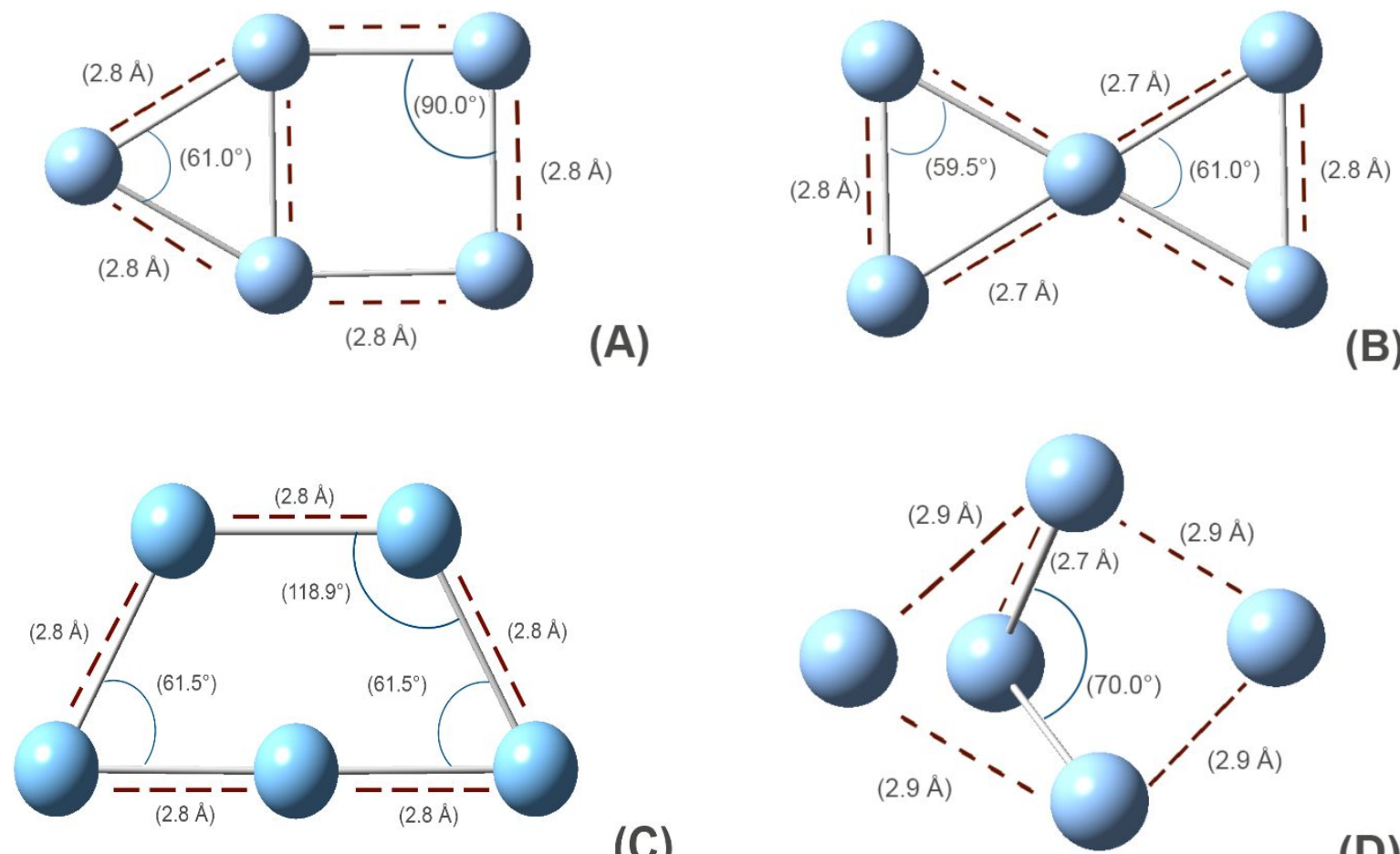

(C)

(D)

Figure S2. Silver conformations converged for the five-silver-atom cluster. (A), (B) and (C) are planar configurations arranged in several possible distributions. (D) is a non-planar 
conformation type with poor structural stability. (C) represents the most stable one, while (D) the least stable.

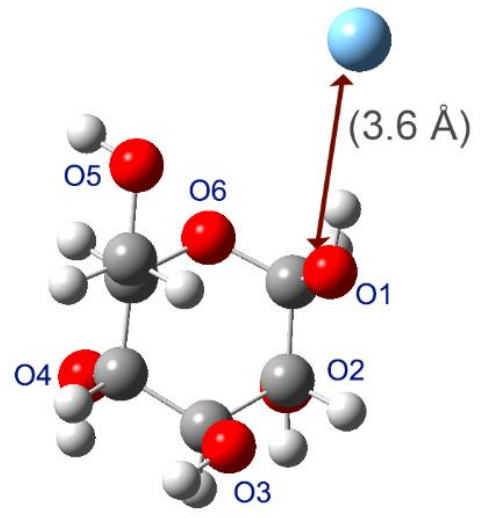

$\Delta$ Eint: -3.2 (A)
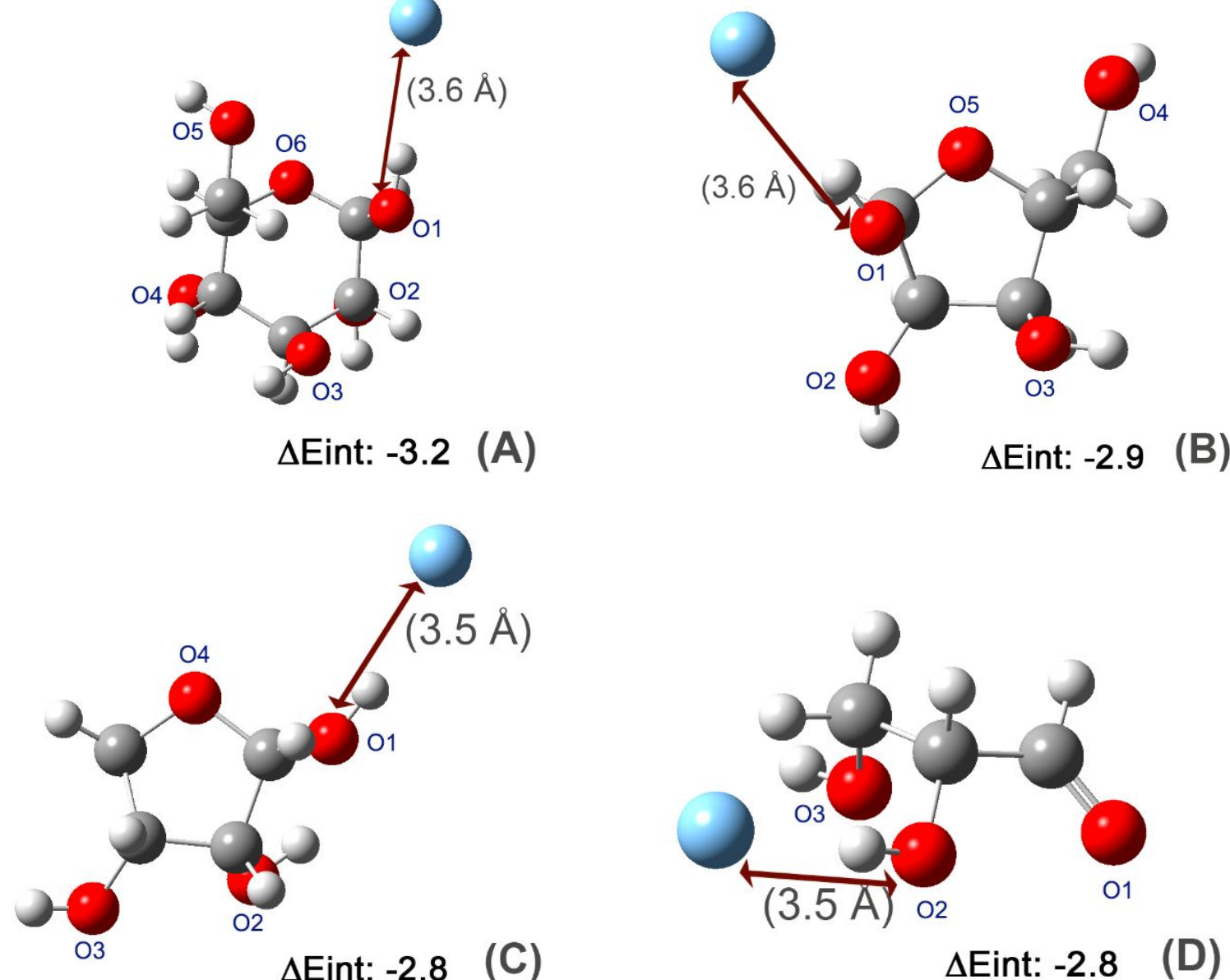

$\Delta$ Eint: $-2.8 \quad(\mathrm{C})$

$\Delta$ Eint: -2.8 (D)

Figure S3. Most stable conformations of one-silver-atom complexed with (A) glucose, (B) ribose, (C) erythrose, and (D) glyceraldehyde molecules. Interaction energies $\left(\Delta \mathrm{E}_{\mathrm{int}}\right)$ are displayed, ranging from -2.8 to $-3.2 \mathrm{kcal} / \mathrm{mol}$; without significant variations between the four complexes. 


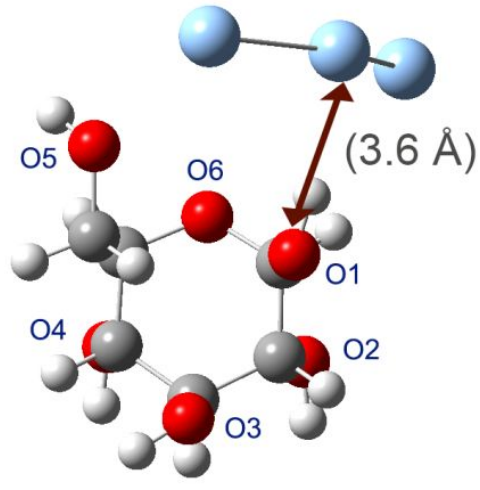

$\Delta$ Eint: -5.4 (A)

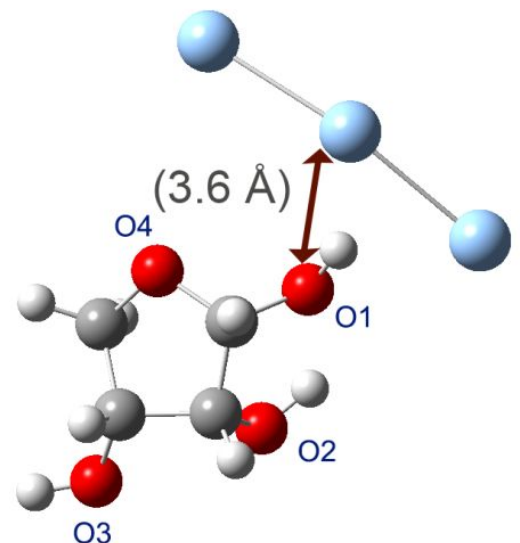

$\Delta$ Eint: $-5.3 \quad(\mathrm{C})$

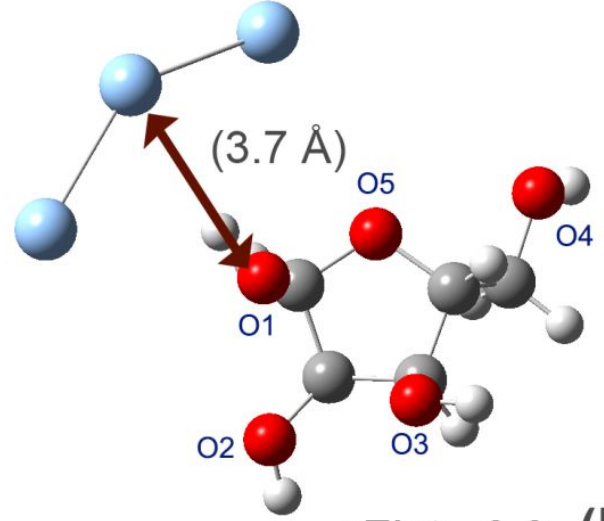

$\Delta$ Eint: -6.6 (B)

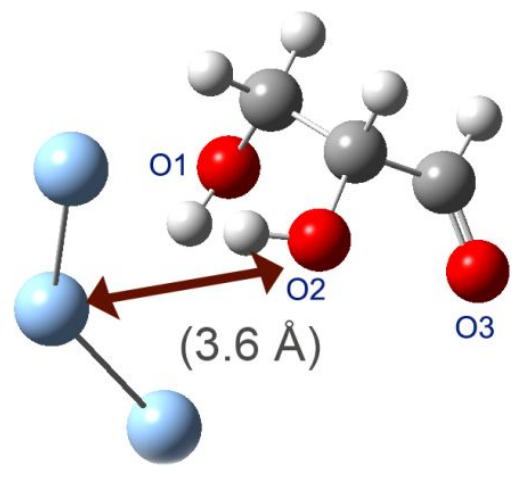

$\Delta$ Eint: -8.4 (D)

Figure S4. Most stable conformations of the three-silver-atom cluster complexed with (A) glucose, (B) ribose, (C) erythrose, and (D) glyceraldehyde molecules. Interaction energies $\left(\Delta \mathrm{E}_{\text {int }}\right)$ with major differences, (D) glyceraldehyde complex with the lowest value, while (A) glucose with the highest. 